\title{
AVALIAÇÃO DO PERFIL HEPÁTICO, DO CONSUMO ALIMENTAR E DE ÁLCOOL EM ESTUDANTES UNIVERSITÁRIOS
}

\section{EVALUATION OF HEPATIC PROFILE, FOOD CONSUMPTION AND ALCOHOL IN UNIVERSITY STUDENTS}

\section{Ana Isabel Conejo Costa $\mathbb{D}^{\mathbb{D}}$, Rafaele Andressa da Silva Belei ${ }^{\mathbb{D}}$, Mariana Aparecida Lopes Ortiz $\mathbb{D}^{\mathbb{D}}$, Larissa Ciupa 10}

Uningá - Centro Universitário Ingá, Maringá/PR, Brasil.

*ciupalarissa@gmail.com

\section{RESUMO}

A esteatose hepática é uma patologia que acomete o fígado e é caracterizada pelo aumento da gordura visceral ocasionando lesões hepáticas. Seu desenvolvimento pode estar associado ao estilo de vida sedentário; à hábitos alimentares não saudáveis; bem como ao uso de tabaco, medicamentos e bebidas alcoólicas, principalmente em jovens universitários devido à alteração de sua rotina. Nesse sentido, o objetivo do trabalho foi avaliar o perfil hepático dos acadêmicos por meio das enzimas TGO, TGP e Gama GT, correlacionando tal perfil com dados obtidos por questionário quanto ao consumo alimentar, ingestão alcoólica, uso de tabaco e a prática de atividade física. Para isso, realizou-se um estudo de corte transversal analítico em indivíduos de ambos os gêneros, com idade entre 18 e 30 anos e de diferentes cursos em que estes acadêmicos preencheram o formulário e realizaram a coleta venosa para as análises laboratoriais. Foram avaliados 46 acadêmicos de diferentes cursos, sendo a maioria do sexo feminino $(67,40 \%)$, solteiros $(93,5 \%)$, sedentários $(73,91 \%)$ e que fazem uso de bebidas alcoólicas $(63,04 \%)$ por pelo menos 3 vezes na semana. Dos acadêmicos analisados, foram $22 \%$ que apresentaram alterações na dosagem sérica das enzimas hepáticas, fato que é relevante e preocupante em se tratando de uma comunidade tão jovem e com fortes indícios negativos quanto ao cuidado com a saúde.

Palavras-chave: Acadêmicos. Álcool. Esteatose. Perfil hepático.

\section{ABSTRACT}

Hepatic steatosis is a condition that affects the liver by increasing the visceral fat causing hepatic damage. The disease development might be associated with sedentary lifestyle; unhealthy eating habits; as well as use of tobacco, medications, and alcoholic beverages especially in college students due to change in their routine. In this sense, the goal of this study was to evaluate the hepatic profile of such students throughout the TGO, TGP and Gamma GT enzymes and to correlate this information with data obtained by a questionnaire regarding food consumption, alcohol intake, tobacco use and physical activity. In order to do so, an analytical cross-sectional study was conducted in individuals of both genders, male and female, aged between 18 and 30 years old and from different graduation courses in which such students filled out a questionnaire form and underwent venous collection for the laboratory analyzes. Forty-six students from different graduation courses were evaluated. Most of them were female $(67.40 \%)$, single $(93.5 \%)$, sedentary $(73.91 \%)$ and making use of alcoholic beverages $(63.04 \%)$ at least 3 times a week. From all the subjects analyzed, were $22 \%$ of them that presented alterations in the serum dosage of liver enzymes, a fact that is relevant and raises our concern because it refers to such a young community with strong negative indications regarding their own health care.

Keywords: Alcohol. College students. Hepatic profile. Steatosis. 


\section{INTRODUÇÃO}

Jovens ao ingressar no curso de ensino superior muitas vezes tem sua rotina afetada pela necessidade de mudar de cidade, morar sozinho, ter responsabilidades, estudar e providenciar sua própria alimentação. Isso faz com que o consumo de alimentos industrializados aumente. $\mathrm{O}$ consumo de bebidas alcoólicas também passa a fazer parte da vida da grande maioria dos acadêmicos e o consumo aumenta conforme o tempo decorrido, sendo mais comum em acadêmicos do sexo masculino (CARDOSO, 2016).

A sociedade atual está exposta a diversos hábitos de risco que afeta a qualidade de vida, a saúde e relacionamentos familiares, entre os fatores de risco se destaca o uso de bebidas alcoólicas e tabaco. Além disso, atualmente se observa um aumento no consumo de alimentos processados e ultra processados o que gera um fator preocupante na alimentação global, pois a qualidade desses alimentos não apresenta uma boa riqueza nutricional (SANTANA; SARTI, 2019; SILVA; ROCHA, 2016).

O fígado é o órgão do corpo humano que é responsável por exercer diversas funções essenciais à vida, sendo elas sintetizar moléculas, armazenar nutrientes que foram absorvidos no trato digestivo, degradar moléculas exógenas, neutralizar e eliminar substâncias tóxicas além de auxiliar na digestão através da produção da bile (HALL, GUYTON, 2011; PINTO, 2012).

Uma patologia que pode acometer o fígado é a esteatose hepática caracterizada pela presença de grandes ou pequenas vesículas de gordura com o predomínio de triglicerídeos acumulados no citoplasma dos hepatócitos. O acúmulo de gordura no fígado pode levar a lesões hepáticas sendo considerada uma das causas mais comum de doenças hepáticas crônicas em países desenvolvidos (PINTO, 2012; KARANJIA, 2016;).

A esteatose tem várias causas, podendo ser uso de medicamentos, ação de agentes tóxicos, algumas anastomoses intestinais para tratamento de obesidade, além de outras condições patológicas, porém a causa mais frequente é a lesão hepática pela ingestão de altas doses de álcool (MINCIS; MINCIS, 2006).

O desenvolvimento da esteatose hepática pode estar associado ao estilo de vida que está cada vez mais atarefado, impossibilitando a prática de atividade física, hábitos alimentares saudáveis e levando ao uso de tabaco, medicamentos, e bebidas alcoólicas (PINTO, 2012).

Marcadores biológicos podem ser usados na triagem básica de disfunção hepática sendo eles a Transaminase Glutâmico Pirúvica (TGP), Transaminase Glutâmico Oxalacética (TGO) e Gama Glutamil transferase (Gama GT). Quando ocorre lesão ou destruição das células hepáticas essas enzimas se encontram aumentadas na corrente sanguínea (JESUS; SOUSA; BARCELOS, 2014).

A localização dessas enzimas auxilia no diagnóstico e prognóstico das doenças hepáticas. A enzima TGP é citoplasmática tendo maior atividade no tecido hepático, já a TGO está localizada tanto em nível citoplasmático quanto em nível mitocondrial, distribuída no músculo esquelético, fígado, coração, rim e cérebro. Quando há lesão tecidual essas enzimas se apresentam aumentadas. A gama GT é encontrada em vários órgãos e tecidos como no fígado, rim, vias biliares, pulmões, intestino, cérebro e coração, sendo utilizada como marcador do sistema biliar (ARAÚJO, 2015).

O presente estudo tem como objetivo avaliar o perfil hepático e o consumo alimentar, tabaco e álcool dos acadêmicos de uma instituição de ensino superior particular.

\section{MATERIAL E MÉTODOS}

Este trabalho foi aprovado pelo comitê de ética 5220 - Faculdade Ingá/ Uningá sob o parecer 3.451.836. Trata-se estudo de corte transversal analítico com acadêmicos, de ambos os gêneros e idade entre 18 e 30 anos, de uma Instituição de Ensino Particular da cidade de Maringá, PR. Da totalidade dos cursos presenciais da área da saúde e exatas foram recrutados aproximadamente 7 indivíduos de cada, totalizando 46 acadêmicos. Os mesmos foram convidados a participar da pesquisa, sendo informados sobre o objetivo do estudo, assinaram o Termo de Consentimento Livre e Esclarecido (TCLE) e responderam a um questionário de frequência de consumo alimentar, 
adaptado de Fonseca, (1999) e de bebidas no mesmo momento em que foi realizado a coleta de sangue venoso no Laboratório de Análises Clínicas Escola da Uningá.

A coleta de sangue venoso foi realizada utilizando sistema simples de captação sanguínea. Foram colhidos $5 \mathrm{ml}$ de sangue, armazenado em tubo sem anticoagulante de separação em gel. Para tal, os participantes do estudo se submeteram a um jejum alimentar de 10 a 12 horas e abstenção de qualquer tipo de bebida alcóolica por um período de 24 horas, assim como preconiza o Ministério da Saúde.

\section{Análise Bioquímica do Perfil hepático}

As amostras foram centrifugadas para se obter o soro, que em seguida foi submetido a dosagens bioquímicas das enzimas TGO, TGP e Gama GT, de forma manual.

Para dosagem da enzima TGO, preparou-se o reagente de trabalho na proporção 4:1, misturando 4 partes do tampão (tampão Tris 121mol/L; L- asparato $362 \mathrm{mmol} / \mathrm{L}$; MDH $460 \mathrm{U} / \mathrm{L}$; $\mathrm{LDH}>660 \mathrm{U} / \mathrm{L}$ e azida sódica 14,6 mmol/L) com uma parte do volume de coenzima (2-cetoglutarato $75 \mathrm{mmol} / \mathrm{L}, \mathrm{NADH} 1,3 \mathrm{mmol} / \mathrm{L}$ e azida sódica $14,6 \mathrm{mmol} / \mathrm{L}$ ). Adicionou-se $1000 \mu \mathrm{L}$ do tampão com $100 \mu \mathrm{L}$ da amostra homogeneizou e incubou em banho-maria a $37^{+} 0,2^{\circ} \mathrm{C}$ por 1 minuto. Registrouse a absorbância inicial e depois em 1, 2 e 3 minutos e registrou a absorbância novamente (ANALISA GOLD®, 2017).

O procedimento do teste para dosar a enzima TGP foi preparar o reagente de trabalho misturando 4 partes do tampão (tampão Tris $150 \mathrm{~mol} / \mathrm{L}$; L- alanina 750 mmol/L; LDH >2300 U/L e azida sódica 14,6 mmol/L) com uma parte do volume de coenzima (2-cetoglutarato $75 \mathrm{mmol} / \mathrm{L}$, NADH 1,3 mmol/L e azida sódica $14,6 \mathrm{mmol} / \mathrm{L}$ ). Adicionou $1000 \mu \mathrm{L}$ do tampão com $100 \mu \mathrm{L}$ da amostra homogeneizou e incubou em banho-maria a $37^{+} 0,2^{\circ} \mathrm{C}$ por 1 minuto. Registrou-se a absorbância inicial e depois em 1, 2 e 3 minutos (ANALISA GOLD®, 2017).

Para dosar a enzima Gama GT foi preparado o reagente de trabalho misturando 4 partes do tampão (glicilglicina $197 \mathrm{mmol} / \mathrm{Le}$ azida sódica 14,6 mmol/L) com uma parte do volume de substrato (gama-glutamil-3-carboxi-4-nitroanilida e azida sódica 14,6 mmol/L). Adicionou-se $1000 \mu \mathrm{L}$ do tampão com $50 \mu \mathrm{L}$ da amostra, homogeneizou e incubou em banho-maria a $37^{+} 0,2^{\circ} \mathrm{C}$ por 1 minuto. Registrou-se a absorbância inicial e depois em 1, 2 e 3 minutos (ANALISA GOLD®, 2018).

Vale ressaltar que a avaliação dos parâmetros bioquímicos apresentados acima emprega-se kits comerciais da marca Analisa Gold ${ }^{\circledR}$ com técnicas padronizadas, baseadas em métodos cinéticos por espectro fotometria seguindo as recomendações do fabricante.

Em todos os testes aos quais os valores foram superiores ao valor de referência, foram realizados em duplicatas para a confirmação do valor encontrado, para garantir a eficiência dos achados.

\section{Análise dos dados}

Todos os dados foram editados em planilhas do Microsoft Excel para que ao final dos procedimentos práticos fossem submetidos a análise estatística, utilizando Bioestat 5.0 e GraphPrism 5 .

\section{RESULTADOS E DISCUSSÃO}

Foram avaliados 46 acadêmicos dos cursos de Agronomia, Biomedicina, Enfermagem, Engenharia Elétrica, Farmácia, Nutrição e Psicologia, na faixa etária de 18 a 30 anos. Em que os participantes com idade entre 18 a 22 anos, $52,2 \%$ eram do sexo feminino e $24,0 \%$ do sexo masculino, em idade entre 23 a 26 anos, $15,2 \%$ eram do sexo feminino e 6,5\% do sexo masculino e com idade entre 27 a 30 anos, 2,2\% do sexo masculino. Dentre as opções de estado civil constatou-se 93,5\% de solteiros e $6,5 \%$ de casados. 


\section{Frequência no consumo de bebida e cigarro e atividade física dos acadêmicos}

Dentre os acadêmicos participantes da pesquisa, a maioria estão no $1 .^{\circ}$ ano de seu respectivo curso. A adesão maior de acadêmicos dos primeiros anos se deve graças à curiosidade, por ser algo novo, pelo tempo disponível. Acadêmicos de anos mais avançados aderiram menos a pesquisa pois muitos tem outras atividades, além das aulas como estágios, projetos de conclusão de curso, o que faz a adesão ser menor.

Tabela 1 - Avaliação da frequência de atividade física, cigarro e bebida de acadêmicos de uma instituição de ensino superior particular de Maringá, Paraná, Brasil.

\begin{tabular}{|c|c|c|c|c|c|c|}
\hline \multirow[t]{3}{*}{ Frequência } & \multicolumn{6}{|c|}{ Variáveis } \\
\hline & \multicolumn{2}{|c|}{ Atividade física } & \multicolumn{2}{|c|}{ Cigarro } & \multicolumn{2}{|c|}{ Bebida } \\
\hline & Feminino & Masculino & Feminino & Masculino & Feminino & Masculino \\
\hline Sim & & & 1 & 3 & 17 & 12 \\
\hline Não & & & 30 & 12 & 14 & 3 \\
\hline Total & & & 31 & 15 & 31 & 15 \\
\hline Todos os dias & & & & & 0 & 0 \\
\hline 1 a 2 vezes & & & & & 3 & 1 \\
\hline Final de semana & & & & & 11 & 7 \\
\hline Uma vez & & & & & 3 & 4 \\
\hline Nenhuma & & & & & 14 & 3 \\
\hline Diariamente & 3 & 3 & & & & \\
\hline 1 a 2 vezes & 10 & 4 & & & & \\
\hline Mais de 3 vezes & 4 & 2 & & & & \\
\hline Não pratica & 14 & 6 & & & & \\
\hline Total & 31 & 15 & & & & \\
\hline
\end{tabular}

Notas: *A frequência para as variáveis considera o tempo de uma semana.

Fonte: os autores.

O ingresso no curso superior, para muitos jovens pode representar o primeiro momento de responsabilidades, a dificuldade de associação das novas tarefas, fatores psicossociais, e estilo de vida são propícios a hábitos inadequados de vida (FREITAS et al., 2013).

Entre os acadêmicos avaliados, observou-se que o consumo de cigarro é de 8,69\% entre os participantes, e este dado considera homens e mulheres. Embora haja diversas campanhas contra o uso do cigarro o hábito de fumar ainda é comum entre os acadêmicos. No estudo de Rosa et al. (2014) observou-se que o consumo de tabaco foi de 8,9\%. De acordo com Munhoz et al. (2017) o tabaco é considerado uma droga, que provoca dependência química, composto por diversos componentes químicos que o torna perigoso para saúde.

Já o consumo de bebidas alcoólicas entre os acadêmicos avaliados foi de 63,04\%, ou seja, mais da metade da população estudada faz uso de bebidas alcoólicas. Quando avaliamos a frequência do consumo se observa que a maioria consome mais aos finais de semana, o que pode ser visto como uma forma de se socializar entre os amigos e diminuir a timidez (NASCIMENTO et al., 2015). Segundo Pechansks (2004), o álcool está entre as substâncias psicoativas mais consumidas pelos jovens o que está relacionada ao seu fácil acesso.

Pesquisas mostram que a longo prazo o consumo excessivo de álcool pode levar a uma série de alterações no fígado, dentre elas a doença hepática alcoólica (BUCHO et al., 2012).

Quanto a pratica de atividade física, 73,91\% dos acadêmicos avaliados diziam não fazer qualquer tipo de atividade física ou faziam esporadicamente. Segundo Marcondelli, Costa e Schmitz (2008), o declínio da prática de atividade física acontece principalmente na adolescência, momento em que também se observa a tendência à obesidade, podendo estar associada à mudança no estilo de vida. 
Segundo Araújo (2015), a associação do exercício físico com alimentação saudável são práticas que interferem diretamente na prevenção de doenças crônicas não transmissíveis.

\section{Qualidade alimentar dos acadêmicos}

A avalição da frequência alimentar mostrou que a alimentação dos jovens nesta fase da vida é mediada pela praticidade, portanto se observa uma qualidade nutricional ruim entre os acadêmicos avaliados.

De acordo com o questionário aplicado observou-se que o alimento mais consumido entre as mulheres é a carne de vaca, balas e doces e fritura (mais de 3x na semana), ao mesmo tempo que tem uma maior ingestão de verdura, legumes e frutas. Já os homens o alimento mais consumido é a carne de vaca, frituras, balas e doces (3x na semana) com uma frequência menor de consumo de verdura, legumes e frutas quando comparado com as mulheres.

Nossos dados corroboram com o estudo de Marcondelli, Costa e Schmitz (2008) que observaram maior consumo de alimentos doces e gordurosos, e redução no consumo de frutas e hortaliças, entre a população estudada.

Em ambos os sexos se observou prevalência do consumo de carne vermelha, que apesar de ser um alimento que deva ser consumido, o excesso da mesma e seus embutidos determinam altas concentrações de colesterol, ácidos graxo insaturados e sódio, podendo ser um fator preocupante (SCHNEIDER; DURO; ASSUNÇÃO, 2014).

Segundo Portela; Melo; Sampaio (2013) a ingestão em excesso de proteínas está associada a resistência à insulina, onde o consumo de carne de vaca e alimentos proteicos, eleva a incidência de diabetes mellitus do tipo II podendo levar à doença hepática gordurosa não alcoólica (DHGNA).

A falta de tempo para preparar o alimento e consumi-lo faz com que muitas vezes a escolha seja por alimentos rápidos e práticos muitas vezes fast food (FRANÇA et al., 2012).

O ambiente universitário, dificulta o hábito da alimentação saudável, pois associa mudança de comportamento, stress, falta de administração do tempo, faz com que os jovens prefiram alimentos rápidos com baixo valor nutricional (LOUREIRO, 2016).

\section{Avaliação Bioquímica do perfil hepático}

Ao avaliar o perfil hepático dos acadêmicos observou-se alterações nas dosagens de TGP e Gama GT em 22\% dos participantes (Tabela 2).

Tabela 2 - Resultado das dosagens séricas dos acadêmicos.

\begin{tabular}{lcccccc}
\hline Dosagens & \multicolumn{2}{c}{ TGO } & \multicolumn{2}{c}{ TGP } & \multicolumn{2}{c}{ GAMA } \\
\cline { 2 - 7 } séricas & Feminino & Masculino & Feminino & Masculino & Feminino & Masculino \\
\hline Normal & 31 & 15 & 30 & 11 & 26 & 14 \\
Elevado & 0 & 0 & 1 & 4 & 5 & 1 \\
\hline
\end{tabular}

Notas: *valores de referência para as dosagens seguem os fornecidos na bula da marca ANALISA GOLD®,2017, pelo método cinético sendo: TGO mulheres 10-37 U/L, homens 11-39 U/L. TGP mulheres 1037 U/L, homens 11-45 U/L. Gama mulheres <40 U/L, homens <60 U/L.

Fonte: ANALISA GOLD®, 2017.

A TGO é encontrada em nível citoplasmático e mitocondrial, identificada no músculo esquelético, cérebro, rim, coração e no fígado e na presença de alteração dos seus níveis séricos pode estar relacionada à lesão em qualquer um dos tecidos (ARAÚJO, 2015). Neste estudo, não se observou alteração na concentração dessa enzima entre os participantes.

A enzima TGP é encontrada no citoplasma dos hepatócitos, e em outros tecidos e órgãos, porém sua maior distribuição é no fígado, sendo assim mais específica para identificar danos hepáticos (FERRAZ; SILVA; SCHIAVON, 2018). Dentre os participantes, observou-se que 4 do 
sexo masculino e 1 do sexo feminino apresentaram valores de TGP aumentado. Com relação ao questionário aplicado o consumo de carne de vaca, frituras é predominante 4 vezes ou mais na semana, e o consumo de verduras, legumes e frutas é de 1 a 3 vezes na semana apenas, a pratica de atividade física é nenhuma ou de 1 a 2 vezes na semana. Além disso, fazem uso de bebida alcoólica.

A enzima Gama GT é encontrada no túbulo proximal renal, fígado, pâncreas e intestino, e níveis séricos de Gama GT estão associados principalmente por dano hepáticos. É uma enzima álcoolinduzida pois níveis maiores de álcool no soro representa uma maior atividade da enzima (ALCÂNTARA, 2007).

Das mulheres avaliadas 5 tiveram os níveis de Gama GT aumentados e relacionando o questionário aplicado pode se observar que de 4 vezes na semana ou mais elas consomem mais carne de vaca, carne salgada e açúcares em contra partida elas consomem verduras, legumes e frutas.

Um participante do sexo masculino apresentou valores elevados de Gama GT, e TGP, a amostra do mesmo se apresentava lipêmica, observa-se também que o mesmo consome 4 vezes ou mais na semana refrigerantes e frituras, e não consome verduras, legumes e frutas, não pratica atividade física e faz o uso de bebidas alcoólicas. Justificando assim uma alimentação com alto índice calórico, e sedentário.

Os resultados observados sugerem o dano hepático, com alteração de TGP e Gama GT em alguns pacientes.

Verifica-se que os acadêmicos que tiveram valores das enzimas alterados apresentam um perfil semelhante, o perfil alimentar tem a ingestão de muita proteína, frituras, doces e pouco consumo de frutas e verduras, o consumo de bebidas alcoólicas é frequente, e a prática de atividade física é esporádica ou nenhuma.

\section{CONCLUSÃO}

Atualmente, pela falta de tempo, as pessoas cada vez mais optam por consumir comidas rápidas, industrializados e alimentos processados, também levam uma vida sedentária, causando diversas doenças modernas que antes não existiam. As consequências desse mal para o organismo são graves e trazem consigo uma série de complicações.

Os dados obtidos no presente trabalho apontam para a gravidade do problema, já que $22 \%$ dos acadêmicos apresentaram alterações nas dosagens bioquímicas. Isso reforça a necessidade da assistência de saúde nesta faixa etária, visando o diagnóstico precoce e principalmente o aconselhamento nutricional, uma vez que a grande maioria dos participantes relataram uma dieta desbalanceada e o uso de álcool como fatores que podemos apontar como fatores de risco para as doenças hepáticas.

\section{REFERÊNCIAS}

ALCÂNTARA, L. I. Avaliação dos níveis de gama-glutamil transpeptidase sérica em pacientes hepatopatas e sua utilização como marcador bioquímico para consumo de álcool. 2007. 65 p. Tese de Doutorado - Universidade de São Paulo, 2007.

ARAÚJO, I. M. S. Alterações hepáticas em acadêmicos do curso de biomedicina do Ceulp/Ulbra. 2015. Monografia - Centro Universitário Luterano de Palmas, Palmas -TO, 2015.

BUCHO, M. S. C. R. et al. Fisiopatologia da doença hepática alcoólica. 2012. Tese de Doutorado - Universidade Fernando Pessoa, Porto, 2012.

CARDOSO, G. A. Consumo alimentar e estilo de vida: Um estudo longitudinal com estudantes universitários. 2016. 202 p. Tese de Doutorado - Universidade de São Paulo, Piracicaba, 2016. 
FERRAZ, M. L. G.; SILVA, A. E. B.; SCHIAVON, J. L. N. Manual de hepatologia para vlínicos e residentes. 1. ed. Rio de Janeiro: Atheneu, 2018.

FONSECA, M. J. M.; CHOR, D.; VALENTE, J. G. Hábitos alimentares entre funcionários de banco estatal: padrão de consumo alimentar. Cadernos de Saúde Pública, v. 15, n. 1, p. 29-39, 1999.

FRANÇA, F. C. O. et al. Mudanças dos hábitos alimentares provocados pela industrialização e o impacto sobre a saúde do brasileiro. I Seminário Alimentação e Cultura na Bahia, Bahia, 2012.

FREITAS, R. W. J. F. et al. Análise do perfil lipídico de uma população de estudantes universitários. Revista Latino-Americana de Enfermagem, v. 21, n. 5, p. 1-8, 2013.

GOLD ANALISA®, Diagnóstico Ltda. ALT-PP. Bolo Horizonte MG, 2017.

GOLD ANALISA®, Diagnóstico Ltda. AST-PP. Belo Horizonte MG, 2017.

GOLD ANALISA®, Diagnóstico Ltda. Gama GT. Belo Horizonte MG, 2018.

HALL, J.E.; GUYTON, A. C. Guyton \& Hall Tratado de Fisiologia Médica. 12. ed. Rio de Janeiro: Elsevier, 2011.

JESUS, G. C.; SOUSA, H. H. B. A.; BARCELOS, R. S. S. Principais patologias e biomarcadores das alterações hepáticas. Estudos, v. 41, n. 3, 2014.

KARANJIA, R. N. et al. Esteatose hepática e fibrose: avaliação não invasiva. Revista Mundial de Gastroenterologia. v. 22, p. 45. 2016.

LOUREIRO, M. P. Estado nutricional e hábitos alimentares de universitários. Segurança Alimentar e Nutricional. v. 23, n. 2, p. 955-972, 2016.

MARCONDELLI, P.; COSTA, T. H. M.; SCHMITZ, B. A. S. Nível de atividade física e hábitos alimentares de universitários do $3 .^{\circ}$ ao $5 .^{\circ}$ semestre da área da saúde. Revista de Nutrição. v. 21 , n. $1,2008$.

MINCIS, M.; MINCIS, R. Enzimas hepáticas: aspectos de interesse prático. Revista Brasileira de Medicina. p. 56-60, 2006.

MUNHOZ, M. P. et al. Incidência de esteatose hepática gordurosa não alcóolica na população adulta atual. Revista Saúde UniToledo, v. 1, n. 2, 2017.

NASCIMENTO, F. G. et al. Consumo de bebidas alcoólicas por estudantes do ensino médio na cidade de Porto Alegre/RS. In: XVII SEMINÁRIO INTERNACIONAL DE EDUCAÇÃO NO MERCOSUL 17,18 e 19 de junho 2015 Anais, 2015.

PECHANSKY, F.; SZOBOT, C. M.; SCIVOLETTO, S. Uso de álcool entre adolescentes: conceitos, características epidemiológicas e fatores etiopatogênicos. Revista Brasileira de Psiquiatria. São Paulo, SP, v. 26, supl. 1, p. 14-17, 2004.

PINTO, C. G. S. et al. Esteatose hepática e estilo de vida ativo: Revisão de literatura. Revista Brasileira de Ciência e Movimento, v. 20, n. 3, p. 125-134, 2012. 
PORTELA, C. L. M.; MELO, M. L. P.; SAMPAIO, H. A. C. Aspectos fisiopatológicos e nutricionais da doença hepática gordurosa não alcoólica (DHGNA). Revista Brasileira de Nutrição Clínica, v. 28, n. 1, p. 54-60, 2013.

ROSA, M. I. et al. Tobacco use and associated factors among students of a university of Criciúma (SC). Cadernos Saúde Coletiva. v. 22, n. 1, p. 25-31, 2014.

SANTANA, A. B. C.; SARTI, F. M. Mapeamento da qualidade nutricional da alimentação em diferentes estados do Brasil. Confins. Revue Franco-Brésilienne de Géographie/Revista FrancoBrasilera de Geografia, n.39, 2019.

SCHNEIDER, B. C.; DURO, S. M. S.; ASSUNÇÃO, M. C. F. Meat consumption by adults in southern Brazil: a population-based study. Ciência \& Saúde Coletiva, v. 19, n. 8, p. 3583-3592, 2014.

SILVA, L. R.; ROCHA, M. S. Avaliação dos marcadores hepáticos em estudantes etilistas da faculdade Anhanguera de Anápolis - Goiás. Revista Acadêmica Oswaldo Cruz. Ano 3, n. 12, 2016. 